\title{
Electrically assisted light-induced azimuthal gliding of the nematic liquid-crystal easy axis on photoaligned substrates
}

\author{
A. V. Dubtsov, ${ }^{1}$ S. V. Pasechnik, ${ }^{1,2}$ Alexei D. Kiselev, ${ }^{3,2, *}$ D. V. Shmeliova, ${ }^{1}$ and V. G. Chigrinov ${ }^{2, \dagger}$ \\ ${ }^{1}$ Moscow State University of Instrument Engineering and Computer Science, Stromynka 20, 107846 Moscow, Russia \\ ${ }^{2}$ Hong Kong University of Science and Technology, Clear Water Bay, Kowloon, Hong Kong \\ ${ }^{3}$ Institute of Physics of National Academy of Sciences of Ukraine, prospekt Nauki 46, 03028 Kiev, Ukraine
}

(Received 30 April 2010; published 2 July 2010)

\begin{abstract}
We study azimuthal gliding of the easy axis that occurs in nematic liquid crystals brought in contact with the photoaligned substrate (initially irradiated azo-dye film) under the action of reorienting UV light combined with in-plane electric field. For irradiation with the linearly polarized light, dynamics of easy axis reorientation is found to be faster as compared to the case of nonpolarized light. Another effect is that it slows down with the initial irradiation dose used to prepare the azo-dye film. This effect is interpreted by using the previously suggested phenomenological model. We present the theoretical results computed by solving the torque balance equations of the model that agree very well with the experimental data.
\end{abstract}

DOI: 10.1103/PhysRevE.82.011702

PACS number(s): $61.30 . \mathrm{Hn}, 42.70 . \mathrm{Gi}$

\section{INTRODUCTION}

It is well known that irradiation of a photosensitive (dye or polymer) layer with linearly polarized UV (LPUV) or visible light may have a profound effect on its properties by producing anisotropy of the angular distribution of molecules. In a liquid crystal (LC) brought in contact with the irradiated layer, the surface ordering originated from the photoinduced anisotropy determines the anchoring characteristics such as the anchoring strengths and the easy axis, $\mathbf{n}_{e}$, directed along the direction of preferential orientation of LC molecules at the surface.

So, in this way, the light can be used as a means to control the anchoring characteristics of photosensitive materials. Technologically, this idea underlies the photoalignment (PA) technique which is employed in the manufacturing process of liquid crystal displays for fabricating high quality aligning substrates [1]. In the PA method, the easy axis is determined by the polarization of the pumping UV light, whereas the azimuthal and polar anchoring strengths may depend on a number of the governing parameters such as the wavelength and the irradiation dose [2].

Macroscopically, overage orientation of LC molecules in interfacial layers is described by the surface director, $\mathbf{n}_{s}$, which is parallel to the easy axis at the equilibrium spatially uniform state. The latter is no longer the case when the orientational structure is deformed by external (electric and/or magnetic) fields. So, in the presence of field-induced director deformations, the surface director may deviate from the easy axis.

It turned out that the easy axis itself may slowly rotate under the action of strong (magnetic or electric) fields. Over the past few decades this slow motion-the so-called easy axis gliding-has received much attention as a widespread phenomenon observed in a variety of liquid crystals on amorphous glass [3], polymer [4-13], and solid [6,11] substrates.

\footnotetext{
*kiselev@iop.kiev.ua

†eechigr@ust.hk
}

Recently, it was found that, in addition to magnetically (electrically) induced gliding, there is the photoinduced gliding of the easy axis in dye-doped liquid crystals $[14,15]$. According to Ref. [15], this effect is driven by the processes of light-induced adsorption/desorption of dye molecules on an aligning polymer surface.

Slow reorientation of the easy axis also takes place on the photosensitive layers prepared using the PA technique. It was originally observed on poly-(vinyl)-alcohol (PVA) coatings with embedded azo-dye molecules [5], whereas similar results for the azo-dye films were reported in Ref. [11]. So, in a LC cell with the initially irradiated layer, subsequent illumination with reorienting light which polarization differs from the one used to prepare the layer can trigger the lightinduced easy axis gliding. Such gliding may be of considerable interest for applications such as LC rewritable devices [16].

In our recent paper [17] we have found that the photoinduced azimuthal gliding of the easy axis on a photoaligned substrate may occur when irradiation with linearly polarized UV light (LPUV) is used in combination with an "in-plane" ac electric field $(f=3 \mathrm{kHz})$ produced by the voltage applied in the lateral direction. This effect might be called the electrically assisted light-induced azimuthal gliding of the easy axis.

In Ref. [17], the emphasis was on slow relaxation of the easy axis back to the initial state after turning off both the electric field and light. This stage of switching off relaxation was controlled by the four parameters: the electric field strength $E$, the light intensity $I$, the exposure time $t_{\text {exp }}$ and the doze $D_{p}$ of the initial UV irradiation. It was found that, at certain combinations of the governing parameters, relaxation considerably slows down (up to several days).

So, the combined effect may be used to tune technical parameters of LC memory devices. Similarly, in the earlier paper [18], an assisting in-plane electric field has been used as a stimulating factor of the light-induced homeotropic-toplanar transition to improve the response sensitivity of the photodriven cells. In addition, it has to be taken into account in applications of LC and PA technique using different field 
combinations that involve intense light beams along with strong electric fields (e.g., in photonics [19]).

In this study azimuthal gliding of the easy axis in the regime of switching on both the in-plane electric field and reorienting (linearly polarized and nonpolarized) UV light (LPUV and NPUV) will be of our primary interest. The layout of the paper is as follows.

In Sec. II, we describe the experimental procedure and setup used to measure the azimuthal easy axis angle as a function of time. The experimental results for the azo-dye films prepared at different initial irradiation doses, $D_{p}$, are presented in Sec. III. In particular, we find that, in the presence of the assisting in-plane electric field, LPUV and NPUV irradiation may equally produce slow changes in LC surface orientation. Another effect is that easy axis reorientation dynamics slows down with the initial irradiation dose, $D_{p}$. In subsection of Sec. III, we apply the phenomenological model of azimuthal gliding [17] to interpret the experimental data measured in the cells with the azo dye layer prepared at different values of $D_{p}$. Finally, in Sec. IV we discuss the results and make some concluding remarks.

\section{EXPERIMENT}

For our measurements, two differently treated glass plates were assembled to prepare the planar LC cell. The cell thickness was $d=17.4 \pm 0.2 \mu \mathrm{m}$. The upper glass plate with a rubbed polyimide film was to provide the strong planar anchoring conditions. The lower one with transparent indium tin oxide (ITO) electrodes and the interelectrode stripes (the gap was $g=50 \mu \mathrm{m}$ ) was covered with a film of photoaligning substance, the azobenzene sulfuric dye SD1 (Dainippon Ink and Chemicals) [1]. The surface of the azo-dye film was irradiated with UV light $(\lambda=365 \mathrm{~nm})$ linearly polarized along the normal to the interelectrode gap so as to produce zones illuminated at different energy exposure doses $D_{p}$ ranged from 0.07 to $0.55 \mathrm{~J} / \mathrm{cm}^{2}$. In this way, there were typically four zones that differ from one another in the initial exposure dose, $D_{p}$, and thus in the initial azimuthal anchoring strength, $W_{0}$. The nematic liquid crystal (NLC) mixture E7 was injected into the cell in an isotropic phase by capillary action and then slowly cooled to room temperature. Note that similar experimental procedure has already been used in our previous study [17].

Experimental setup was based on a polarized microscope connected with a fiber optics spectrometer and a digital camera, where rotating polarizer technique was used to measure the azimuthal angle $\phi_{e}$ characterizing orientation of the easy axis. The measurements were carried out at a temperature of $26{ }^{\circ} \mathrm{C}$.

We used the irradiation of a UV lamp of $6 \mathrm{~W}$ power at the wavelength $\lambda=365 \mathrm{~nm}$ (absorption spectrum for SD1 has a peak at $365 \mathrm{~nm}$ ) as a reorienting light beam which, as is shown in Fig. 1, was normally impinging onto the bottom substrate coated with the azo-dye layer. Referring to Fig. 1, for LPUV irradiation, the polarization vector of UV light, $\mathbf{E}$, was directed along the initial orientation of LC molecules, $\mathbf{n}_{0}$, which is parallel to the electrode stripes. So, LPUV light and the electric field make the LC molecules align in the same direction.

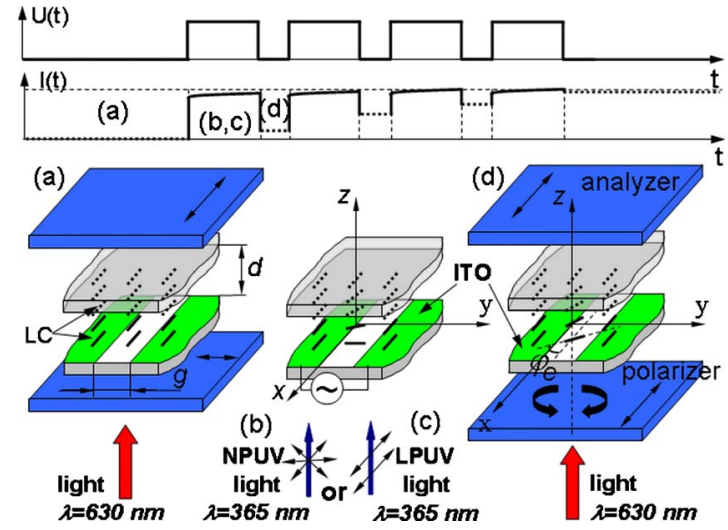

FIG. 1. (Color online) Geometry of the experiment: (a) initial state; (b) switching on an in-plane electric field combined with NPUV irradiation; (c) switching on an in-plane electric field combined with LPUV irradiation; (d) switching off the electric field and light to measure the easy axis azimuthal angle $\phi_{e}$.

Each measurement was conducted on a fresh area of the cell, protected from the negative irradiation. In order to register microscopic images and to measure the value of the easy axis azimuthal angle, $\phi_{e}$, the electric field and light were switched off for about $1 \mathrm{~min}$. This time interval is short enough (dynamics at surface is considerably slower than that in the bulk) to ensure that orientation of the easy axis remains essentially intact in the course of measurements.

\section{RESULTS}

Figure 2 shows the microscopic images obtained at different times of irradiation with LPUV and NPUV reorienting light. In this case, the in-plane ac voltage $(U=100 \mathrm{~V}, f=3 \mathrm{kHz})$ and the initial irradiation dose $\left(D_{p}=0.27 \mathrm{~J} / \mathrm{cm}^{2}\right)$ are kept constant. Note that, when applying sufficiently strong electric field $(E=2 \mathrm{~V} / \mu \mathrm{m})$ in combination with reorienting irradiation for more than $30 \mathrm{~min}$, memory effects take place. It means that, after switching off the field and light, the easy axis did not relax back to its initial state for at least few months.

When an in-plane ac electric field is applied to the transparent ITO electrodes, twist-like deformations of LC director

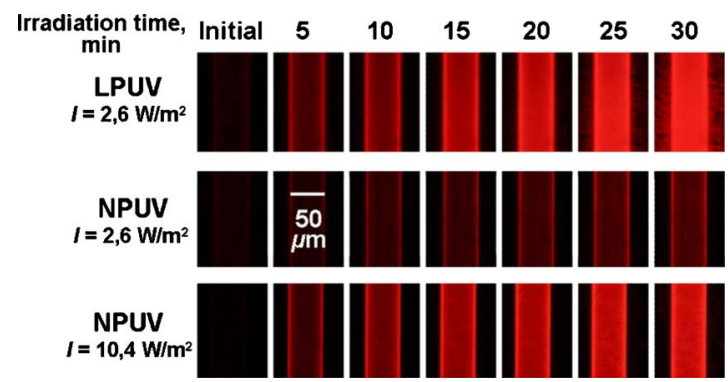

FIG. 2. (Color online) Microscopic images of the cell (filter with $\lambda=630 \mathrm{~nm}$ was used) in crossed polarizers for LPUV and NPUV irradiation at different irradiation times. The interelectrode gap $(g=50 \mu \mathrm{m})$ is indicated. The ac electric field is $E=2 \mathrm{~V} / \mu \mathrm{m}$ and the initial irradiation dose is $D_{p}=0.27 \mathrm{~J} / \mathrm{cm}^{2}$. 


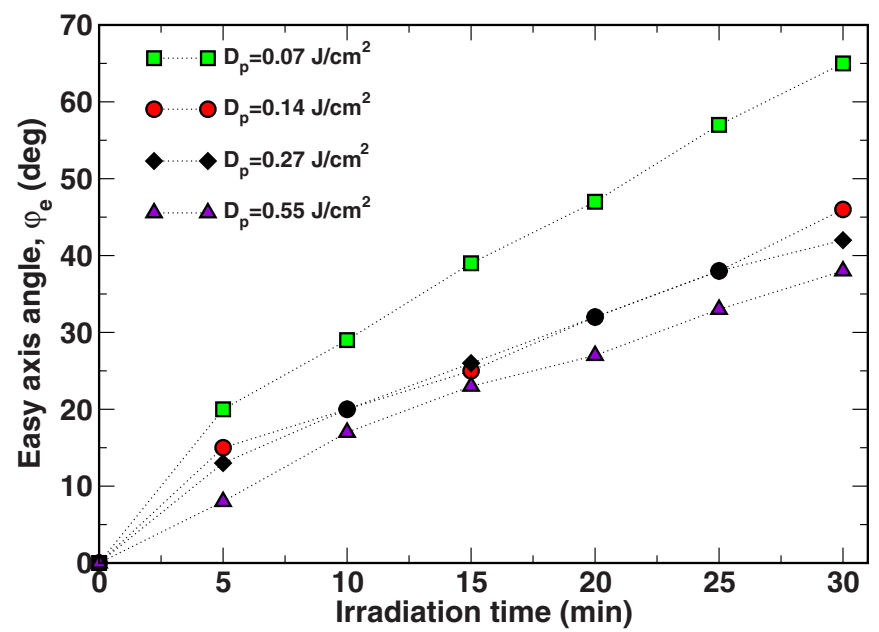

FIG. 3. (Color online) Easy axis angle as a function of the irradiation time measured in the samples prepared at different initial irradiation doses $D_{p}$. The reorienting UV light is nonpolarized with $\lambda=365 \mathrm{~nm}$ and $I=10.4 \mathrm{~W} / \mathrm{m}^{2}$; the applied voltage is $U=100 \mathrm{~V}$.

occur. In crossed polarizers, such deformations reveal themselves as the bright stripes that can be seen in Fig. 2 .

Given the light intensity $I=2.6 \mathrm{~W} / \mathrm{m}^{2}$, reorientation dynamics at LPUV irradiation is considerably faster as compared to the case of NPUV light. In order to produce variations of optical picture similar to those for LPUV light, the intensity of NPUV irradiation had to be increased at least in four times from $2.6 \mathrm{~W} / \mathrm{m}^{2}$ to $10.4 \mathrm{~W} / \mathrm{m}^{2}$. This can be seen from Figs. 3 and 4, where the easy axis angle $\phi_{e}$ is plotted against the irradiation time at different initial irradiation doses $D_{p}$ for NPUV and LPUV light, respectively.

According to Figs. 3 and 4, reorientation of the easy axis slows down with the initial irradiation dose $D_{p}$ that determines the initial value of the azimuthal anchoring strength. In [2], this anchoring strength was found to be an increasing

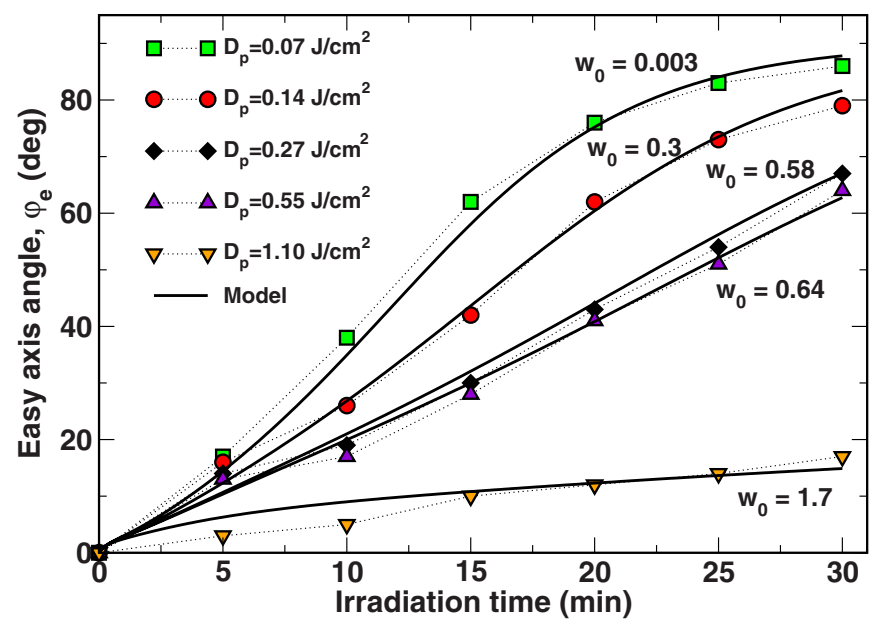

FIG. 4. (Color online) Easy axis angle versus the irradiation time at different initial irradiation doses $D_{p}$. The reorienting UV light is linearly polarized with $\lambda=365 \mathrm{~nm}$ and $I=2.6 \mathrm{~W} / \mathrm{m}^{2}$; the applied voltage is $U=100 \mathrm{~V}$. Solid lines represent the theoretical curves computed at the indicated initial values of the anchoring parameter $w_{0}=W_{0}(0)(\xi-h) /\left(2 K_{22}\right)$. function of the dose $D_{p}$. So, the lower the initial azimuthal anchoring strength the faster gliding is.

\section{Model}

We can now apply the phenomenological model formulated in $[11,17]$ to describe this effect. According to this model, the anchoring characteristics of the photoaligned layer such as the easy axis, $\mathbf{n}_{e}$, are determined by orientational order of LC molecules adsorbed by the azo-dye film. The adsorbed molecules can be divided into two ensembles characterized by two mutually orthogonal directions of preferential orientation of long molecular axes: $\mathbf{n}_{0}$ and $\mathbf{n}_{2}$, $\mathbf{n}_{0} \perp \mathbf{n}_{2}$. Interestingly, this assumption bears a resemblance to the dual-axis models previously employed to study competitive effects of photoalignment and microgrooves [20] and to describe the anchoring transitions on substrates irradiated with light and a plasma beam $[21,22]$.

Ordering of the first ensemble is governed by the orientational order parameter of the azo dye layer induced by the initial irradiation with linearly polarized UV light (PA treatment made before the cell is filled with LC). The direction of preferential orientation, $\mathbf{n}_{0}$, is dictated by the azo-dye order parameter which is known to depend on the irradiation dose $D_{p}$ [2]. So, the initial number of LC molecules in this ensemble $N_{0}(0)$ and the initial anchoring strength $W_{0}(0)$ are determined by the initial irradiation dose $D_{p}$.

When the cell filled with LC is irradiated by the reorienting light with polarization normal to the one used at the preparation stage for PA treatment of the azo-dye layer, absorbed LC molecules undergo light-induced transitions to the second ensemble aligned along the vector, $\mathbf{n}_{2}$, orthogonal to $\mathbf{n}_{0}$. These transitions occur due to reorientation of dye molecules interacting with the adsorbed LC layer. Since the anchoring strengths, $W_{0}$ and $W_{2}$, for the easy axes of the ensembles, $\mathbf{n}_{0}$ and $\mathbf{n}_{2}$, are proportional to the corresponding numbers of molecules $N_{0}$ and $N_{2}: W_{0,2} \propto N_{0,2}$, it can be concluded that the strength $W_{0}\left(W_{2}\right)$ decreases (increases) with irradiation time.

The second ensemble can also be populated by applying an in-plane electric field in the direction $\mathbf{n}_{2}$. It can be explained in terms of adsorption-desorption processes taking place in the near-surface layer of characteristic thickness $h$. Initially, in the absence of electric field, the surface director $\mathbf{n}_{s}$ characterizing average orientation of LC molecules in the near-surface layer is directed along the easy axis $\mathbf{n}_{0}$. So, the adsorption-desorption processes do not influence the undisturbed angular distributions of LC molecules in the absorbed and near-surface layers which are initially identical.

An electric field $\mathbf{E}$ produces a twist deformation on the distance $\xi$ defined as the electric coherence length,

$$
\xi=\frac{1}{E} \sqrt{K_{22} /\left(\varepsilon_{0} \Delta \varepsilon\right)}
$$

where $\Delta \varepsilon$ is the electric permittivity anisotropy, $K_{22}$ is the Frank elastic constant for the twist deformation. The distance $\xi$ decreases with $E$ down to the values comparable with the thickness of the near-surface layer $h$. In our case, the electric field is $E=U / d \approx 2 \mathrm{~V} / \mu \mathrm{m}$ and, for the liquid crystal mixture 
E7 with the twist elastic constant $K_{22} \approx 6.5 \times 10^{-12} \mathrm{~N}$ and the dielectric anisotropy $\Delta \varepsilon \approx 13.7$, the electric coherence length $\xi$ can be estimated at about $0.12 \mu \mathrm{m}$.

Under this electric-field-induced deformation, the surface director, $\mathbf{n}_{s}$, inclines toward the direction $\mathbf{n}_{2}\left(\mathbf{E} \| \mathbf{n}_{2}\right.$ and $\Delta \varepsilon$ $>0)$. The absorption-desorption processes involving exchange of molecules between the differently aligned (absorbed and near-surface) layers will eventually produce reorientation of absorbed molecules along the direction of the electric field. Note that, owing to low probability of adsorption-desorption events, noticeable changes may require very long periods of time. In terms of the model, this can be described an increase in the number of molecules $\mathrm{N}_{2}$ and in the anchoring strength $W_{2}$.

Though the mechanisms behind slow reorientation of adsorbed LC molecules induced by light and electric field are essentially different, in the phenomenological model $[11,17]$, they can be treated along similar lines. In particular, orientation of the easy axis $\mathbf{n}_{e}$ characterized by the azimuthal angle $\varphi_{e}$ is defined by the balance of the two torques arising from the two ensembles $N_{0}$ and $N_{2}$ : the torque transmitted from the bulk by the near-surface layer and the viscous torque proportional to the specific viscosity of gliding $\gamma_{e}$. For the surface director $\mathbf{n}_{s}$ with the azimuthal angle $\varphi_{s}$, the analogs balance involves the torque arising due to deviation of the surface director from the easy axes (it is proportional to the surface anchoring energy strength $W_{s}$ ), the torque transmitted from the bulk and the viscous torque proportional to the surface viscosity $\gamma_{s}$. The resulting system of equations for the easy axis and surface director azimuthal angles, $\varphi_{e}$ and $\varphi_{s}$, was derived in [17] and can be conveniently written in the following dimensionless form:

$$
\begin{aligned}
& \frac{\partial \varphi_{e}}{\partial \tau}=\left[\pi / 2-\varphi_{s}\right]-w_{e} \sin 2 \varphi_{e}, \quad \tau=t / \tau_{e}, \\
& \frac{\partial \varphi_{s}}{\partial \tau}=\gamma\left\{\left[\pi / 2-\varphi_{s}\right]-w_{s} \sin 2\left(\varphi_{e}-\varphi_{s}\right)\right\},
\end{aligned}
$$

where $\tau_{e}=\gamma_{e}(\xi-h) / K_{22}$ is the characteristic time of easy axis reorientation; $\gamma=\gamma_{e} / \gamma_{s}$ is the viscosity ratio; $w_{e, s}=W_{e, s}(\xi$ $-h) /\left(2 K_{22}\right)$ is the dimensionless anchoring parameter and $W_{e}=W_{0}-W_{2}$ is the effective anchoring parameter which defines the strength of coupling between the easy axis $\mathbf{n}_{e}$ and the initial state of surface orientation described by the vector $\mathbf{n}_{0}$.

From the above discussion, the initial value of the easy axis anchoring parameter (coupling strength) $W_{e}$ is determined by the initial irradiation doze $D_{p}$ and equals $W_{0}(0)$. In the course of irradiation with reorienting light assisted by an in-plane electric field, the anchoring parameter $W_{e}$ declines and can even become negative. The latter indicates that the direction of preferential orientation at the azo-dye film is changed from $\mathbf{n}_{0}$ to $\mathbf{n}_{2}$.

For the purposes of modeling, the exponential dependence

$$
W_{e}=W_{0}(0)-A_{L}\left(1-e^{-t / \tau_{L}}\right)-A_{E}\left(1-e^{-t / \tau_{E}}\right),
$$

where the characteristic times $\tau_{L}\left(\tau_{E}\right)$ and the amplitudes $A_{L}\left(A_{E}\right)$ are related to the action of light (electric field), can be regarded as a reasonable approximation.

Before making comparison between the model and experiment, we shall dwell briefly on the general properties of the dynamical system [Eq. (2)]. Since the gliding viscosity $\gamma_{e}$ is typically several orders higher than the surface viscosity $\gamma_{s}$, the viscosity ratio is large, $\gamma \gg 1$. In this case, in the initial stage of reorientation process, the surface director angle, $\varphi_{s}$, changes abruptly reaching the adiabatic regime. In this regime, the temporary evolution of the angles is subject to the linking relation,

$$
\pi / 2-\varphi_{s}=w_{s} \sin 2\left(\varphi_{e}-\varphi_{s}\right)
$$

So, the easy axis angle, $\varphi_{e}$, can be conveniently expressed in terms of the surface director angle, $\varphi_{s}$, as follows:

$$
\varphi_{e}=\pi k_{\nu}+\frac{\pi}{4}(\nu+1)-\left(\psi_{s}+\frac{\nu}{2} \arcsin \left[\psi_{s} / w_{s}\right]\right),
$$

where $\psi_{s}=\pi / 2-\varphi_{s}, \nu= \pm 1$ and $k_{\nu} \in \mathbb{Z}$ is the integer. Then substituting the relation (5) into Eq. (2a) gives the dynamic equation,

$$
\begin{gathered}
\frac{\partial \psi_{s}}{\partial \tau}=\frac{\sqrt{w_{s}^{2}-\psi_{s}^{2}}}{\sqrt{w_{s}^{2}-\psi_{s}^{2}}+\nu / 2} F_{\nu}\left(\psi_{s}\right), \\
F_{\nu}=w\left(\psi_{s} \cos 2 \psi_{s}+\nu \sqrt{w_{s}^{2}-\psi_{s}^{2}} \sin 2 \psi_{s}\right)-\psi_{s},
\end{gathered}
$$

where $w=W_{e} / W_{s}$ is the anchoring ratio.

The equilibrium states characterized by the azimuthal angles, $\varphi_{e}^{(s t)}$ and $\varphi_{s}^{(s t)}$, can be found as the stable (attracting) stationary points of the dynamical system [Eq. (2)]. It is not difficult to show that, at $\left|w_{s}\right|<0.5$, the surface director equilibria are among the zeros of the function, $F_{\nu}$, given in Eq. (7). More specifically, for $\psi_{s}^{(s t)}=\pi / 2-\varphi_{s}^{(s t)}$, we have

$$
F_{\nu}\left(\psi_{s}^{(s t)}\right)=0, \quad \nu F_{\nu}^{\prime}\left(\psi_{s}^{(s t)}\right)<0
$$

The equilibrium conditions [Eq. (8)] can now be combined with the expression (7) to yield the dependence of the surface director angle equilibria on the anchoring ratio, $w=W_{e} / W_{s}$, in the following parametrized form:

$$
\varphi_{s}^{(s t)}(w)=\left\{\begin{array}{l}
w \equiv w_{\nu}(t), \\
\varphi_{s}^{(s t)}=\pi / 2+t, \quad-\left|w_{s}\right| \leq t \leq\left|w_{s}\right|,
\end{array}\right.
$$

where $w_{\nu}(t)=\left[\cos 2 t+\nu \sqrt{w_{s}^{2}-t^{2}} \sin (2 t) / t\right]^{-1}$.

Figure 5 shows the bifurcation curves that are evaluated using Eq. (9) and describe dynamical behavior of the model in relation the anchoring ratio.

It is seen that there is the only stationary value of the surface director angle, $\varphi_{s}^{(s t)}=\pi / 2$, provided that $w<w_{+}$or $w>w_{-}$, where $w_{ \pm}=w_{ \pm}(0)=1 /\left(1 \pm 2 w_{s}\right)$ is the critical (bifurcation) value of the anchoring ratio. The equilibrium values of the easy axis angle in these two regions are: $\varphi_{e}^{(s t)}=\pi / 2$ at $w<w_{+}$and $\varphi_{e}^{(s t)}=0$ at $w>w_{-}$. It means that, in the case of sufficiently large coupling parameter $W_{e}$, where $w>w_{-}$, the easy axis gliding is completely suppressed, whereas, in the 


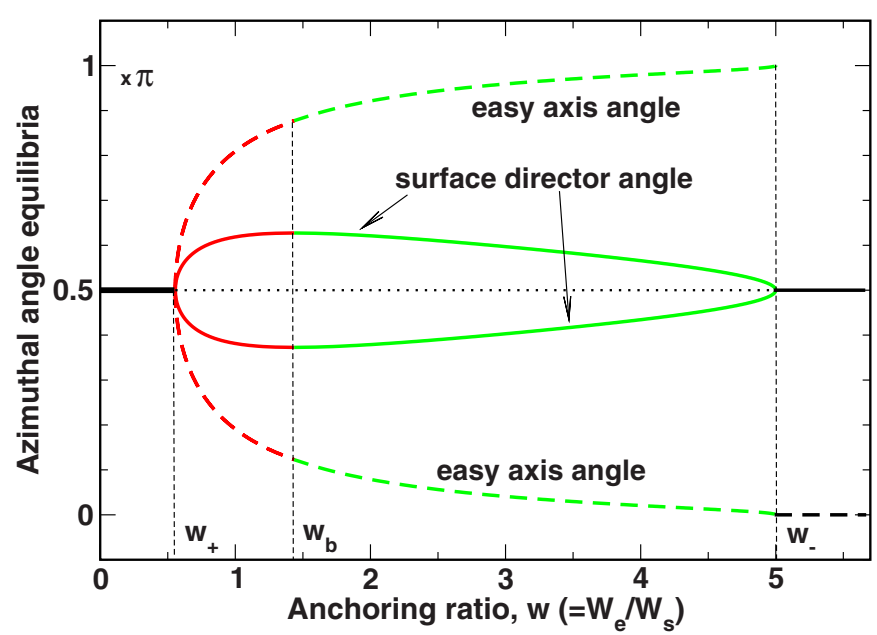

FIG. 5. (Color online) Bifurcation diagram for equilibria of easy axis and surface director azimuthal angles computed from Eq. (9) as a function of the anchoring ratio, $w=W_{e} / W_{s}$, at $w_{s}=0.4$.

regime of weak coupling with $w<w_{+}$, the easy axis rotates approaching the stationary state of the surface director, $\mathbf{n}_{s}^{(s t)}$ $=\mathbf{n}_{2}$.

As it can be seen in Fig. 5, when the anchoring ratio $w$ passes through the critical points $w_{ \pm}$the pitchfork bifurcations [23] occur and the stationary state $\varphi_{s}^{(s t)}=\pi / 2$ becomes unstable. So, for the surface director angle in the intermediate region with $w_{+}<w<w_{-}$, there are two symmetrically arranged stable stationary points: $\varphi_{s}^{( \pm)}=\pi / 2 \pm \Delta \varphi_{s}^{(s t)}$. In this case, gliding is not suppressed, but, by contrast to the regime of weak coupling, the equilibrium states of the easy axis and the surface director are no longer identical.

Referring to Fig. 5, there is also the branching point, $w_{p}$ $=w_{ \pm}\left(w_{s}\right)=1 / \cos \left(2 w_{s}\right)$, in this region. At $w<w_{p}\left(w>w_{p}\right)$, the equilibria are defined by the zeros of the function $F_{+}\left(F_{-}\right)$. Note that the magnitude of the equilibrium angle $\psi_{s}^{(s t)}$ reaches its maximum, $\left|\psi_{s}^{(s t)}\right|=\left|w_{s}\right|$, at $w=w_{p}$. In the vicinity of these extrema, relaxation of both surface director and easy axis angles will slow down. It follows from the expression on the right hand side of dynamical Eq. (6) which is proportional to $\sqrt{w_{s}^{2}-\psi_{s}^{2}}$.

In order to apply the model to interpret experimental data, the system [Eq. (2)] has to be solved numerically. In Fig. 4, it is demonstrated that by using the model we can obtain the theoretical results which are in good agreement with the experimental data.

The parameters used for computing the curves are: $\tau_{e}=10 \mathrm{~min}, \gamma=100$ and $w_{s}=0.45$. Since, at $E=2 \mathrm{~V} / \mu \mathrm{m}$, $K_{22} / \xi \approx 5.4 \times 10^{-5} \mathrm{~J} / \mathrm{m}^{2}$, the azimuthal anchoring strength $W_{s}$ can be estimated at $2.4 \times 10^{-5} \mathrm{~J} / \mathrm{m}^{2}$. For the easy axis anchoring parameter $w_{e}$, we assumed the simplified exponential dependence $w_{e}=w_{0}-\Delta w\left(1-e^{-\tau / \tau_{w}}\right)$ with $\Delta w=1$ and $\tau_{w}=2$. The only parameter that depends on the initial dose is the initial value of the easy axis anchoring parameter $w_{0}=w_{e}(0)$. The values of $w_{0}$ are shown in Fig. 4: $w_{0}=0.003\left(W_{0} \approx 1.6 \times 10^{-7} \mathrm{~J} / \mathrm{m}^{2}\right)$ at $D_{p}=0.07 \mathrm{~J} / \mathrm{cm}^{2}$; $w_{0}=0.3\left(W_{0} \approx 1.6 \times 10^{-5} \mathrm{~J} / \mathrm{m}^{2}\right) \quad$ at $\quad D_{p}=0.14 \mathrm{~J} / \mathrm{cm}^{2}$; $w_{0}=0.58\left(W_{0} \approx 3.1 \times 10^{-5} \mathrm{~J} / \mathrm{m}^{2}\right) \quad$ at $\quad D_{p}=0.27 \mathrm{~J} / \mathrm{cm}^{2}$; $w_{0}=0.64\left(W_{0} \approx 3.5 \times 10^{-5} \mathrm{~J} / \mathrm{m}^{2}\right)$ at $D_{p}=0.55 \mathrm{~J} / \mathrm{cm}^{2}$; and
$w_{0}=1.7\left(W_{0} \approx 9.2 \times 10^{-5} \mathrm{~J} / \mathrm{m}^{2}\right)$ at $D_{p}=1.1 \mathrm{~J} / \mathrm{cm}^{2}$. So, typical azimuthal anchoring strengths and the above estimates for $W_{s}$ and $W_{0}$ are of the same order. As is expected, the initial easy axis anchoring parameter $W_{0}$ increases with the dose $D_{p}$. Interestingly, at $D_{p}=0.55 \mathrm{~J} / \mathrm{cm}^{2}$, the estimate for the characteristic time $\gamma_{e} / W_{0}=\tau_{e} / w_{0} \approx 937 \mathrm{~s}$ is close to the value, $\gamma_{e} / W_{0} \approx 880 \mathrm{~s}$, reported in Ref. [17] for the liquid crystal mixture ZhK 616.

\section{DISCUSSION AND CONCLUSIONS}

In this paper we have studied electrically assisted photoinduced gliding of the NLC easy axis on the photoaligned azo-dye layer. Our experimental results indicate that, in the presence of sufficiently strong in-plane electric field, LPUV and NPUV light can both be effective in reorienting the easy axis. But, for NPUV irradiation, the intensity of the pumping beam should be several times higher than the intensity of LPUV light.

We have also found that reorientation dynamics of the easy axis depends on the initial dose of UV irradiation used to prepare the azo-dye film. Reorientation is retarded appreciably as the dose increases. So, it comes as no surprise that the initial irradiation counteracts easy axis gliding induced by the secondary irradiation with differently polarized reorienting light.

It is shown that this effect can be described by using the phenomenological model previously suggested in Refs. $[11,17]$. The model was formulated as the dynamical system of the balance torque Eqs. (2) for the easy axis and the surface director azimuthal angles. Its dynamical behavior governed by the ratio of the easy axis and surface director anchoring parameters, $w=W_{e} / W_{s}$, is characterized by two pitchfork bifurcations. The bifurcation points, $w_{+}$and $w_{-}$, are at the boundaries of the regions with $w<w_{+}$and $w>w_{-}$ where dynamics of easy axis reorientation occurs in the regimes of weak and strong coupling, respectively. At $w<w_{+}$ the easy axis rotates toward the equilibrium state with $\varphi_{e}^{(s t)}=\varphi_{s}^{(s t)}=\pi / 2$, whereas, at $w>w_{-}$, reorientation is suppressed and $\mathbf{n}_{e}^{(s t)}=\mathbf{n}_{0}\left(\varphi_{e}^{(s t)}=0\right)$.

We have fitted the experimental data by numerically solving the system [Eq. (2)]. Despite the fact that good agreement between the computed curves and the data (see Fig. 4) counts in favor of the model, its theoretical justification has yet to be done. In particular, it is still to be clarified how the model can be applied to the case of irradiation with nonpolarized light. For such irradiation, by contrast to linearly polarized actinic light, there is no polarization vector related to an in-plane direction of preferred orientation of molecules in the azo-dye layer and the only symmetry axis (the direction of light propagation) is normal to the substrates. So, in this case, the reorienting light has a destructive effect on the initial photoinduced ordering.

In the language of the model, the light-induced degradation of the ordering can be described as a decrease of the number of adsorbed molecules in the first ensemble. Hence it enhances (and facilitates) the action of the electric field which alone populates the second ensemble leading to an increase in the corresponding anchoring strength. 
This mechanism, in agreement with our experimental results, implies that the efficiency of nonpolarized light should be considerably lower as compared to the case of linearly polarized light. Note that a detailed analysis of the initial ordering decay that underlies electrically assisted gliding induced by nonpolarized light may be complicated by a number of factors such as out-of-plane reorientation. Such analysis is beyond the scope of this paper.

A deeper insight into complicated molecular mechanisms behind the gliding under consideration requires additional experimental studies and a more systematic theoretical treat- ment of the processes involved. Such treatment has to deal with the interplay of photoinduced ordering in azo-dye films [24] and the adsorption-desorption processes $[4,15,25,26]$ underlying the gliding phenomenon.

\section{ACKNOWLEDGMENTS}

This work was partially supported by grants: Development of the Higher Schools Scientific Potential 2.1.1/5873; Grant No. NK-410P; HKUST CERG RPC07/08.EG01 and CERG Grant Nos. 612208 and 612409.
[1] V. G. Chigrinov, V. M. Kozenkov, and H.-S. Kwok, Photoalignment of Liquid Crystalline Materials: Physics and Applications, Series in Display Technology (Wiley, Chichester, 2008), p. 219.

[2] A. D. Kiselev, V. G. Chigrinov, and D. D. Huang, Phys. Rev. E 72, 061703 (2005).

[3] E. A. Oliveira, A. M. F. Neto, and G. Durand, Phys. Rev. A 44, R825 (1991).

[4] P. Vetter, Y. Ochmura, and T. Uchida, Jpn. J. Appl. Phys., Part 2 32, L1239 (1993).

[5] V. P. Vorflusev, H.-S. Kitzerow, and V. Chigrinov, Appl. Phys. Lett. 70, 3359 (1997).

[6] S. Faetti, M. Nobili, and I. Raggi, Eur. Phys. J. B 11, 445 (1999).

[7] I. Jánossy and T. I. Kósa, Phys. Rev. E 70, 052701 (2004).

[8] S. Joly, K. Antonova, P. Martinot-Lagarde, and I. Dozov, Phys. Rev. E 70, 050701(R) (2004).

[9] S. Faetti and P. Marianelli, Phys. Rev. E 72, 051708 (2005).

[10] S. Faetti and P. Marianelli, Liq. Cryst. 33, 327 (2006).

[11] S. V. Pasechnik, V. G. Chigrinov, D. V. Shmeliova, V. A. Tsvetkov, V. N. Kremenetsky, L. Zhijian, and A. V. Dubtsov, Liq. Cryst. 33, 175 (2006).

[12] O. Buluy, A. Iljin, E. Ouskova, Y. Reznikov, C. Blanc, M. Nobili, and K. Antonova, J. Soc. Inf. Disp. 14, 603 (2006).

[13] I. Jánossy, Phys. Rev. E 81, 031714 (2010).

[14] D. Statman, V. Basore, Y. Sulai, B. Dunlap, and I. Janossy, Liq. Cryst. 35, 33 (2008).

[15] D. Fedorenko, K. Slyusarenko, E. Ouskova, V. Reshetnyak, K. R. Ha, R. Karapinar, and Y. Reznikov, Phys. Rev. E 77, 061705 (2008).
[16] A. Muravsky, A. Murauski, V. Chigrinov, and H.-S. Kwok, Jpn. J. Appl. Phys. 47, 6347 (2008).

[17] S. V. Pasechnik, A. V. Dubtsov, D. V. Shmeliova, V. A. Tsvetkov, and V. G. Chigrinov, Liq. Cryst. 35, 569 (2008).

[18] L. Cui, P. Xie, R. Zhang, and T. Yang, Liq. Cryst. 26, 1541 (1999).

[19] S. V. Pasechnik, V. G. Chigrinov, and D. V. Shmeliova, Liquid Crystals: Viscous and Elastic Properties (Wiley-VCH, Berlin, 2009), p. 424.

[20] D.-H. Chung, T. Fukuda, Y. Takanishi, K. Ishikawa, H. Matsuda, H. Takezoe, and M. A. Osipov, J. Appl. Phys. 92, 1841 (2002).

[21] D. Andrienko, Y. Kurioz, Y. Reznikov, and Y. Reshetnyak, Sov. Phys. JETP 112, 2045 (1997).

[22] O. V. Yaroshchuk, A. D. Kiselev, and R. M. Kravchuk, Phys. Rev. E 77, 031706 (2008).

[23] J. Guckenheimer and P. Holmes, Nonlinear Oscillations, Dynamical Systems, and Bifurcations of Vector Fields, Applied Mathematical Sciences (Springer-Verlag, New York, 1990), Vol. 42, p. 459.

[24] A. D. Kiselev, V. G. Chigrinov, and H.-S. Kwok, Phys. Rev. E 80, 011706 (2009).

[25] E. Ouskova, Y. Reznikov, S. V. Shiyanovskii, L. Su, J. L. West, O. V. Kuksenok, O. Francescangeli, and F. Simoni, Phys. Rev. E 64, 051709 (2001).

[26] G. Barbero and L. R. Evangelista, Adsorption Phenomena and Anchoring Energy in Nematic Liquid Crystals, The Liquid Crystals Book Series (Taylor \& Francis Group, LLC, Boca Raton, 2006), p. 352. 